\title{
Cisto ovariano em vacas de leite: incidência, resposta à aplicação de GnRH e desempenho reprodutivo
}

[Ovarian cysts in lactating dairy cows: incidence, response to GnRH, and reproductive performance]

\author{
R.M. Santos ${ }^{1}$, D.G.B. Démetrio ${ }^{2}$, J.L.M. Vasconcelos $^{3}$ \\ ${ }^{1}$ Faculdade de Medicina Veterinária - UFU \\ Av. Pará, 720 \\ 38400-902 - Uberlândia, MG \\ ${ }^{2}$ Médica veterinária autônoma \\ ${ }^{3}$ Faculdade de Medicina Veterinária e Zootecnia - UNESP - Botucatu, SP
}

\begin{abstract}
RESUMO
A incidência de cistos ovarianos, a resposta ao tratamento com GnRH e os efeitos da ocorrência de cisto no desempenho reprodutivo e na taxa de descarte foram determinados em vacas lactantes da raça Holandesa. Vacas lactantes $(n=333)$ foram avaliadas semanalmente por ultrassonografia a partir da quarta semana pósparto, visando à detecção de corpos lúteos $(\mathrm{CL})$ e de folículos ovarianos maiores que $10 \mathrm{~mm}$. Na sétima semana pós-parto, as vacas foram classificadas: em ciclando $(n=248$; presença de CL em um dos exames ultrassonográficos); em anestro $(n=54$; ausência de $C L$ e de folículos $>25 \mathrm{~mm}$ ) e com cisto ( $n=31$; ausência de $\mathrm{CL}$ e presença de estruturas $>25 \mathrm{~mm}$ ), quando foram distribuídas em: grupo-controle ( $\mathrm{n}=16$; sem tratamento) e grupo-tratamento ( $n=15$; vacas que receberam uma aplicação de $\mathrm{GnRH})$. A taxa de cura foi de $60,0 \%$ no grupo das vacas tratadas e de $87,5 \%$ no grupo-controle. As vacas com cistos apresentaram maior intervalo partoprimeira inseminação artificial $(\mathrm{P}<0,05 ; 91,4 \pm 8,3$ vs. 77,8 $\pm 2,5)$, maior número de serviços por concepção

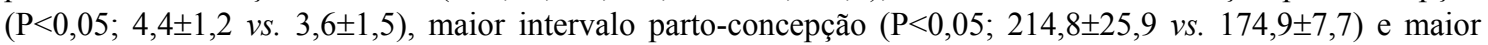
taxa de descarte $(\mathrm{P}<0,05 ; 41,2$ vs. $21,8 \%)$ do que as vacas ciclando. Vacas diagnosticadas com cisto na sétima semana pós-parto apresentaram recuperação espontânea, embora o intervalo parto-concepção e a taxa de descarte tenham sido maiores para essas vacas.
\end{abstract}

Palavras-chave: vacas de leite, eficiência reprodutiva, cisto ovariano, GnRH

\begin{abstract}
The incidence of ovarian cysts, response to GnRH treatment, and effects on reproductive performance and culling rate of Holstein cows were determined. Ovaries of lactating cows $(n=333)$, were weekly monitored by ultrasound, beginning at fourth week postpartum, to determine the presence of corpus luteum (CL) and follicles greater than $10 \mathrm{~mm}$. In the seventh week the cows were classified as cycling ( $n=248$; presence of corpus luteum (CL) in one of the ultrasound evaluations); anovulatory ( $n=54$; absence of $C L$ and follicles less than 25mm), and cystic ( $n=31$; absence of $C L$ and presence of structures greater than 25mm). The cysts cows were distributed in two groups in the seventh week: control group $(n=16$; without treatment $)$ and treatment group ( $n=15$; cows received one GnRH injection). The recovery rate was $60.0 \%$ in treated cows and $87.5 \%$ in control cows. The cystic cows had longer average interval from parturition to first $A I(P<0.05 ; 91.4 \pm 8.3$ vs. 77.8 \pm 2.5$)$, higher number of services per conception $(P<0.05 ; 4.4 \pm 1.2$ vs. 3.6 \pm 1.5$)$, longer interval parturition to conception $(P<0.05 ; 214.8 \pm 25.9$ vs. 174.9 \pm 7.7$)$, and higher percentage of culled cows $(P<0.05 ; 41.2 \%$ vs. $21.8 \%$ ), than cycling cows. Cystic cows without treatment had the same recovery rate as cows treated with GnRH. Cystic cows had longer intervals from parturition to conception, and higher culling rate.
\end{abstract}

Keywords: dairy cows, reproduction efficiency, ovarian cysts, GnRH

Recebido em 6 de outubro de 2008

Aceito em 8 de abril de 2009

E-mail: ricasantos@famev.ufu.br 


\section{INTRODUÇÃO}

Em bovinos, a ocorrência de cisto ovariano é definida como presença de uma estrutura anovulatória de diâmetro maior do que $25 \mathrm{~mm}$ que persiste por, no mínimo, 10 dias na ausência de um corpo lúteo (Garverick, 1997).

Os cistos ovarianos são classificados em folicular ou lúteo, dependendo do grau de luteinização da estrutura. A diferenciação por palpação retal é difícil e às vezes impossível (Sprecher et al., 1988; Farin et al., 1990), entretanto a dosagem de progesterona ( $\mathrm{P} 4)$ no plasma ou no leite pode ajudar a diferenciar os dois tipos de cistos. Vacas com cisto folicular apresentaram concentração plasmática de P4 menor que $1 \mathrm{ng} / \mathrm{mL}$, e vacas com cisto lúteo, acima de $1 \mathrm{ng} / \mathrm{mL}$ (Leslie e Bosu, 1983). A ultrassonografia também pode ser usada para fazer essa diferenciação, fato constatado por Farin et al. (1990), que obtiveram com o ultrassom a sensibilidade de $91,5 \%$ para diagnosticar cisto lúteo e $70 \%$ para cisto folicular.

A ocorrência de cistos relatada em estudos que usavam a palpação retal como forma de diagnóstico foi de 6 a 19\% (Kesler e Gaverick, 1982; Gaverick, 1997). Em estudos mais recentes, a incidência da condição anovulatória (detectada por dosagem hormonal ou ultrassonografia) em vacas de leite varia entre 18 a 19\% (Cartmill et al., 2001; Gümen et al., 2003). Mais de $70 \%$ dos cistos ovarianos ocorrem entre 16 a 50 dias após o parto, com ocorrência mais alta entre 30 a 40 dias (Erb e White, 1981; Kirk et al., 1982). Nanda et al. (1991) observaram a ocorrência de cistos ovarianos em vacas de 20 a 150 dias após o parto. A ocorrência de cistos ovarianos influencia o intervalo de partos, pois causa aumento de 22 a 64 dias no período de serviço (Erb e Martin, 1980; Lee et al., 1988). Savio et al. (1990) relataram que vacas com cistos e vacas normais apresentaram intervalo parto-primeira ovulação de 58 e 12 dias, respectivamente.

A possível causa do cisto ovariano ainda não é bem conhecida. Inúmeros fatores são apontados como fatores de risco, como perda de escore de condição corporal no pós-parto, número de lactações, época do ano e desordens do pós-parto
(López-Gatius et al., 2002). O desenvolvimento do cisto parece estar associado a um desequilíbrio endócrino envolvendo o eixo hipotálamo-hipófise-gonadas (Kesler e Garverick, 1982; Bosu e Peter, 1987). Gümen et al. (2002) e Gümen e Wiltbank (2005a) relataram que a formação de um folículo ovariano grande, similar a um cisto folicular, pode ser induzida pela aplicação de estradiol, que induz o pico de GnRH e LH, na ausência da luteinização subsequente, seja por aplicação de estradiol na ausência de um folículo dominante (Gümen et al., 2002) ou por remoção do corpo hemorrágico (Gümen e Wiltbank, 2005a). Assim, indução do novo pico de GnRH e LH pelo estradiol ficaria inibida devido à necessidade da exposição à progesterona para que esse novo pico possa ser induzido pelo estradiol.

O tratamento do cisto depende da classificação em cisto folicular ou lúteo. Se for folicular, o objetivo é luteinizá-lo com tratamento com gonadotrofina coriônica humana (hCG) ou com $\mathrm{GnRH}$, que induz a onda pré-ovulatória de $\mathrm{LH}$, tratamentos que parecem ter sucesso em $80 \%$ dos casos (Osawa et al., 1995; Garverick, 1997). A prostagladina F2 $\alpha$ (PGF2 $\alpha$ ) causa lise do cisto lúteo ou do cisto folicular luteinizado sete dias após o tratamento com GnRH ou hCG (Cantley et al., 1975). Estudos sugerem que o tratamento com GnRH mais dispositivo vaginal de P4 durante sete dias, seguido de aplicação de PGF2 $\alpha$ ao final do tratamento, é o mais eficaz (Thatcher et al., 1993; Gümen e Wiltbank, 2005b; Kim et al., 2006; Todoroki e Kaneko, 2006), provavelmente devido à etiologia dos cistos relacionada com deficiência de exposição a P4 (Gümen et al., 2002; Gümen e Wiltbank, 2005a) e à baixa concentração de $\mathrm{P} 4$ apresentada por vacas de leite de maior produção, devido à alta ingestão de matéria seca (Santos e Vasconcelos, 2006).

É difícil avaliar o sucesso do tratamento, uma vez que pelo menos $20 \%$ dos cistos têm recuperação espontânea (Kesler e Garverick, 1982; Dobson e Nanda, 1992; López-Gatius et al., 2002).

Este estudo teve como objetivos avaliar: a porcentagem de vacas ciclando, em anestro ou com cisto na sétima semana após o parto; o 
efeito da época do ano na incidência dos cistos; a resposta das vacas com cisto ao tratamento com uma aplicação de GnRH; a porcentagem de recuperação espontânea dos cistos; a eficiência reprodutiva e a taxa de descarte de vacas que apresentaram cisto.

\section{MATERIAL E MÉTODOS}

No período de abril a janeiro, foram avaliadas 333 vacas, de um rebanho de aproximadamente 600 vacas em lactação, da raça Holandesa, com produção média de $8.700 \mathrm{~kg}$ de leite por lactação de 305 dias, mantidas em free stalls, alimentadas com dieta total composta de silagem de milho, feno de alfafa, polpa cítrica, grãos de milho, farinha de soja, minerais e vitaminas.

Os ovários das vacas foram submetidos a avaliações ultrassonográficas semanais, a partir da quarta semana após o parto (22 a 28 dias), para a detecção da presença de corpo lúteo (CL) ou folículos com diâmetro superior a $10 \mathrm{~mm}$. Após as quatro primeiras avaliações (sétima semana após o parto), as vacas foram assim classificadas: em ciclando (presença de CL em um dos exames ultrassonográficos); em anestro (ausência de CL e de estruturas maiores que $25 \mathrm{~mm}$ ) e em vacas com cisto (ausência de CL e presença de estruturas maiores que $25 \mathrm{~mm}$ ).

Na sétima semana após o parto (43 a 49 dias), as vacas císticas $(n=31)$ foram distribuídas em dois grupos: grupo-controle $(\mathrm{n}=16)$, formado por vacas com cisto e que não receberam tratamento, e grupo-tratamento $(n=15)$, formado por vacas com cistos e que receberam uma aplicação de GnRH (100mcg de gonadorelina, Cystorelin ${ }^{\circledR}$, $2 \mathrm{~mL}$, via intramuscular).

Após a divisão dos grupos e a aplicação do $\mathrm{GnRH}$, os animais foram avaliados em mais dois momentos (oitava e nona semana após o parto) para verificar a ocorrência de ovulação ou luteinização e a presença de um novo CL ou de novos folículos.

Posteriormente, foram analisados os dados do intervalo parto-primeira inseminal artificial (IA), do número de serviços por concepção, do intervalo parto-concepção e da taxa de descarte, para avaliar os efeitos da presença do cisto na eficiência reprodutiva.
O efeito da época do ano na incidência de cistos foliculares e o do tratamento na recuperação dos cistos foram analisados pela teoria dos modelos lineares generalizados, que inclui o Modelo de Bernouille para proporções. A influência da ocorrência de cistos no intervalo do partoprimeira-IA, no número de serviços por concepção e no intervalo do parto-concepção foi submetida à análise de variância (SAS/1988).

\section{RESULTADOS E DISCUSSÃO}

A incidência de vacas classificadas como ciclando e que apresentavam estruturas ovarianas com mais de $25 \mathrm{~mm}$ de diâmetro na presença de CL foi de 3.9\% (13/333). A porcentagem de vacas com cistos na sétima semana após o parto foi de 9,3\% (Tab. 1), o que se assemelha aos resultados apresentados por Garverick (1997) e Lopéz-Gatius et al. (2002) que relataram incidência entre 7 a 13,1\% em vacas de leite.

Tabela 1. Classificação de vacas da raça Holandesa na sétima semana após o parto, de acordo com quatro avaliações ovarianas prévias, por ultrassonografia

\begin{tabular}{lc}
\multicolumn{1}{c}{ Classificação } & $\mathrm{N}(\%)$ \\
\hline Vacas ciclando $^{1}$ & $248(74,5)$ \\
Vacas em anestro $^{2}$ & $54(16,2)$ \\
Vacas com cisto $^{3}$ & $31(9,3)$ \\
\hline Total & $333(100)$ \\
\hline
\end{tabular}

${ }^{1}$ Presença de CL em uma das avaliações.

${ }^{2}$ Ausência de CL em todas as avaliações e de estruturas maiores do que $25 \mathrm{~mm}$ de diâmetro.

${ }^{3}$ Ausência de CL em todas as avaliações e presença de estruturas maiores do que $25 \mathrm{~mm}$ de diâmetro.

Foi detectado efeito $(\mathrm{P}<0,05)$ de época do ano na incidência de cistos foliculares (Tab. 2), observando-se que a porcentagem de vacas com cistos foi maior nos meses de abril a julho, provavelmente devido ao fato de as vacas que apresentaram cistos terem parido nos meses mais quentes do ano, quando os problemas relacionados ao parto e o estresse são mais altos. Segundo Bosu e Peter (1987), o aumento do nível de cortisol nas doenças do pós-parto está ligado à ocorrência de cistos. Lopéz-Gatius et al. (2002) também obtiveram dados confirmadores à ideia de que as vacas que parem no verão têm 2,6 vezes mais chances de desenvolver cisto até o dia 49 após o parto do que as vacas que parem no inverno. 
Tabela 2. Época do ano sobre a incidência de cistos em vacas da raça Holandesa na sétima semana após o parto

\begin{tabular}{lcc}
\multicolumn{1}{c}{ Época do ano } & $\begin{array}{c}\text { Número de } \\
\text { vacas }\end{array}$ & $\begin{array}{c}\text { Número de vacas } \\
\text { com cisto }(\%)\end{array}$ \\
\hline Abril/Julho & 174 & $24(13,8)$ \\
Agosto/Outubro & 84 & $5(6,0)$ \\
Novembro/Janeiro & 75 & $2(2,7)$ \\
\hline Total & 333 & $31(9,3)$ \\
\hline
\end{tabular}

${ }^{\mathrm{T}}$ Ausência de corpo lúteo e presença de estruturas maiores do que $25 \mathrm{~mm}$ de diâmetro, em quatro avaliações ultrassonográficas prévias.

Depois do tratamento, na sétima semana após o parto, as vacas foram novamente avaliadas na oitava e na nona semana para verificar a resposta dos cistos ao tratamento e a taxa de cura espontânea. Não foi detectado efeito de tratamento. Por conseguinte, a taxa de cura foi de $60,0 \%(9 / 15)$ no grupo das vacas tratadas e de $87,5 \%(14 / 16)$ no grupo-controle.

Os resultados de cura espontânea foram acima dos citados pela literatura. Estudos apontam que $20 \%$ dos cistos têm recuperação espontânea (Bierschwal et al., 1975; Kesler e Garverick, 1982; Dobson e Nanda, 1992). Morrow et al. (1966) relataram 50\% de taxa de recuperação espontânea em vacas nos primeiros 60 dias pósparto, e Dinsmore et al. (1989) concluíram que vacas em início de lactação não apresentam alta taxa de cura espontânea dos cistos. López-Gatius et al. (2002) apontaram que a taxa de cura espontânea dos cistos, nos primeiros 60 dias pósparto, depende do número de lactações e observaram que $80 \%$ dos cistos das vacas de primeira lactação tiveram cura espontânea, com uma taxa de $30 \%$ nas vacas com mais lactações.

Foram detectadas diferenças entre vacas com cisto, em anestro e ciclando nas características reprodutivas avaliadas. As vacas com cistos apresentaram maior $(\mathrm{P}<0,05)$ intervalo partoprimeira IA, maior número de serviços por concepção e maior intervalo parto-concepção que vacas ciclando (Tab. 3).

Tabela 3. Intervalo do parto-primeira IA, número de serviços por concepção e intervalo do partoconcepção em vacas Holandesas classificadas como ciclando, em anestro e com cisto, na sétima semana após o parto, de acordo com quatro avaliações ovarianas prévias, por ultrassonografia

\begin{tabular}{lccc}
\multicolumn{1}{c}{ Classificação } & $\begin{array}{c}\text { Intervalo parto- } \\
\text { primeira IA (d) }\end{array}$ & $\begin{array}{c}\text { Serviços por } \\
\text { concepção (n) }\end{array}$ & $\begin{array}{c}\text { Intervalo parto- } \\
\text { concepção }(\mathrm{d})\end{array}$ \\
\hline Vacas ciclando* & $77,8 \pm 2,5 \mathrm{a}$ & $3,6 \pm 1,5 \mathrm{a}$ & $174,9 \pm 7,7 \mathrm{a}$ \\
Vacas em anestro** & $94,9 \pm 3,1 \mathrm{~b}$ & $3,7 \pm 1,7 \mathrm{a}$ & $181,5 \pm 10,3 \mathrm{a}$ \\
Vacas com cisto*** & $91,4 \pm 8,3 \mathrm{~b}$ & $4,4 \pm 1,2 \mathrm{~b}$ & $214,8 \pm 25,9 \mathrm{~b}$ \\
\hline
\end{tabular}

Valores seguidos por letras distintas na coluna diferem entre si $(\mathrm{P}<0,05)$.

${ }^{1}$ Presença de CL em uma das avaliações.

${ }^{2}$ Ausência de CL em todas as avaliações e de estruturas maiores do que $25 \mathrm{~mm}$ de diâmetro.

${ }^{3}$ Ausência de CL em todas as avaliações e presença de estruturas maiores do que $25 \mathrm{~mm}$ de diâmetro.

Os resultados de desempenho reprodutivo assemelham-se com os apresentados por outros pesquisadores ao observarem que os cistos influenciam o intervalo de partos por aumentarem de 22 a 64 dias no período de serviço (Erb e Martin, 1980; Lee et al., 1988). Segundo Carroll et al. (1990), o número de dias do parto à primeira IA tende a ser maior, mas a concepção e o intervalo parto-concepção não são afetados pela ocorrência de cistos. Douthwaite e Dobson (2000) reportaram que, após o tratamento, a fertilidade das vacas com cistos foliculares é semelhante à das outras vacas do rebanho, e que as vacas com cisto lúteo apresentam intervalo de parto 40 dias maior.

As vacas com cisto apresentaram taxa de descarte, por problemas diversos, maior $(\mathrm{P}<0,05)$ do que as vacas ciclando $(41,2$ vs. $21,8 \%)$. Douthwaite e Dobson (2000) também verificaram taxa de descarte maior para vacas com cistos foliculares (41 vs. 11\%). 
O desempenho reprodutivo das vacas que apresentaram cisto na sétima semana após o parto ficou comprometido provavelmente devido ao atraso no retorno à ciclicidade, o que aumentou $\mathrm{o}$ intervalo parto-primeira IA, o intervalo parto-concepção e a taxa de descarte.

\section{CONCLUSÃO}

Vacas diagnosticadas com cisto na sétima semana após o parto apresentaram recuperação espontânea, embora o intervalo parto-concepção e a taxa de descarte tenham sido maiores para essas vacas.

\section{REFERÊNCIAS BIBLIOGRÁFICAS}

BIERSCHWAL， C.J.; GARVERICK, H.A.; MARTIN, C.E. et al. Clinical response of dairy cows with ovarian cysts following treatment with GnRH. J. Anim. Sci., v.41, p.1660-1665, 1975

BOSU, W.T.K.; PETER, A.T. Evidence for a role of intrauterine infections in pathogenesis of cysts ovaries in postpartum dairy cows. Theriogenology, v.28, p.725-736, 1987

CANTLEY, T.C.; GARVERICK, H.A.; BIERSCHWAL, C.J. et al Hormonal responses of dairy cows with ovarian cysts to GnRH. $J$. Anim. Sci., v.41, p.1666-1673, 1975.

CARROLL, D.J.; PIERSON, R.A.; HAUSER, E.R. et al. Variability of ovarian structures and plasma progesterone profiles in dairy cows with ovarian cysts. Theriogenology, v.34, p.349-370, 1990

CARTMILL, J.A.; EL-ZARKOUNY; S.Z.; HENSLEY, B.A. et al. Stage of cycle, incidence, and timing of ovulation, and pregnancy rates in dairy cattle after three timed breeding protocols. J. Dairy Sci., v.84, p.1051-1059, 2001.

DINSMORE, R.P.; WHITE, M.E.; GUARD, C.L. et al. Effect of gonadotropin-releasing hormone on clinical response and fertility in cows with cysts ovaries, as related to milk progesterone concentration an days after parturition. J. Am. Vet. Med. Assoc., v.195, p.327-330, 1989.

DOBSON, H.; NANDA, A.S. Reliability of cyst diagnosis and effect of energy status on $\mathrm{LH}$ released by estradiol or GnRH in cows with ovarian cysts. Theriogenology, v.37, p.465-472, 1992

DOUTHWAITE, R.; DOBSON, H. Comparison of different methods of diagnosis of cystic ovarian disease in cattle and an assessment of its treatment with a progesterone-releasing intravaginal device. Vet. Rec., v.23, p.355-259, 2000 .

ERB, H.N.; MARTIN, S. W. Interrelationships between production and reproductive diseases in Holstein cows. J. Dairy Sci., v.63, p.1911, 1980.

ERB, N.H.; WHITE, M.E. Incidence rates of cysts follicles in Holstein cows according to 15 days and 30 days intervals. Cornell Vet., v.71, p.326-331, 1981.

FARIN, P.W.; YOUNGQUIST, R.S.; PARFET, J.R. et al. Diagnosis of luteal and follicular cysts in dairy cows by sector scan ultrasonography. Theriogenology, v.34, p.633-643, 1990

GARVERICK, H.A. Ovarian follicular cysts in dairy cows. J. Dairy Sci., v.80, p.995-1004, 1997

GÜMEN, A.; GUENTHER, J.N.; WILTBANK, M.C. Follicular size and response to Ovsynch versus detection of estrus in anovular and ovular lactating dairy cows. J. Dairy Sci., v.86, p.31843194, 2003.

GÜMEN, A.; SARTORI, R.; COSTA, F.M. et al. A GnRH/LH surge without subsequent progesterone exposure can induce development of follicular cysts. J. Dairy Sci., v.85, p.43-50, 2002.

GÜMEN, A.; WILTBANK, M.C. Follicular cysts occur after a normal estradiol-induced GnRH/LH surge if the corpus hemorrhagicum is removed. Reproduction, v.129, p.737-745, 2005a.

GÜMEN, A.; WILTBANK, M.C. Length of progesterone exposure needed to resolve large follicle anovular condition in dairy cows. Theriogenology, v.63, p.202-218, 2005b.

KESLER, D.J.; GARVERRICK, H.A. Ovarian cysts in dairy cattle: A review. J. Anim. Sci., v.55, p.1147-1159, 1982.

KIM, I.H.; SUH, G.H.; KIM, U.H. et al. A CIDR-based timed AI protocol can be effectively used for dairy cows with follicular cysts. Anim. Reprod. Sci., v.95, p.206-213, 2006. 
KIRK, J.H.; HUFFMAN, E.M.; LANE, M. Bovine cysts ovarian disease. Hereditary relationship and case study. J. Anim. Vet. Assoc., v.181, p.474-476, 1982

LEE, L.A.; FERGUSON, J.D.; GALLIGAN, D.T. The use of survival analysis to quantitative days open: advantages and applications. Acta. Vet. Scand., v.84, suppl., p.433-435, 1988.

LESLIE, D.J.; BOSU, W.T.K. Plasma progesterone concentrations in dairy cows with cystic ovaries and clinical response following treatment with fenoprostalene. Can. Vet. J., v.24, p.352-356, 1983.

LÓPEZ-GATIUS, F.; SANTOLARIA, P.; YÁNIZ, J. et al. Risk factors for postpartum ovarian cysts and their spontaneous recovery or persistence in lactating dairy cows. Theriogenology, v.58, p.1623-1632, 2002.

MORROW, D.A.; ROBERT, S.J.; McENTEE, $\mathrm{K}$. et al. Postpartum ovarian activity and uterine involution in dairy cattle. J. Am. Vet. Med. Assoc., v.149, p.1596-1609, 1966.

NANDA, A. S.; WARD, W.R.; DOBSON, H. Lack of LH response to oestradiol treatment in cows with cystic ovarian disease and effect of progesterone treatment or manual rupture. Res. Vet. Sci., v.51, p.180-184, 1991.

OSAWA, T.; NAKAO, T.; KIMURA, M. et al. Fertirelin and Burserelin compared by release, milk progesterone and subsequent reproductive performance in dairy cows treated for follicular cysts, Theriogenology, v.44, p.835-847, 1995.

SANTOS, R.M.; VASCONCELOS, J.L.M. Ingestão de concentrado e concentração plasmática de progesterona em vacas da raça Holandesa. Arq. Bras. Med. Vet. Zootec., v.58, p.1162-1167, 2006.

SAVIO, J. D.; BOLAND, M.P.; HYNES, N. et al. Resumption of follicular activity in the early postpartum period of dairy cows. J. Reprod. Fertil., v.88, p.569-579, 1990.

SPRECHER, D.J.; NEBEL, R.L.; WHITTIER, W.D. Predictive value of palpation per rectum versus milk and serum progesterone levels for diagnosis of bovine follicular and luteal cysts. Theriogenology, v.30, p.701-710, 1988

THATCHER, W.W.; DROST, M.; SAVIO, J.D. et al. New clinical uses of GnRH and its analogues in cattle. Anim. Reprod. Sci., v.33, p.27-49, 1993

TODOROKI, J.; KANEKO, H. Formation of follicular cysts in cattle and therapeutic effects of controlled internal drug release. J. Reprod. Dev., v.52, p.1-11, 2006.

USER'S Guide: Statistics, Version 6.02. Cary, NC: SAS Institute, 1988. 